\title{
A SUBNORMAL TOEPLITZ COMPLETION
}

\section{In Sung Hwang, An Hyun Kim and Jongrak LeE}

Abstract. In this paper we deal with a subnormal Toeplitz completion problem: Complete the unspecified Toeplitz operators of the partial block Toeplitz matrix

$$
G:=\left[\begin{array}{cc}
U^{* p} & ? \\
? & U^{* q}
\end{array}\right] \quad(p, q=1,2, \cdots)
$$

to make $G$ subnormal, where $U$ is the shift on the Hardy space $H^{2}(\mathbb{T})$ of the unit circle $\mathbb{T}$.

Mathematics subject classification (2010): Primary 47B20, 47B35, 46J15, 15A83; Secondary 30H10, 47A20.

Keywords and phrases: Toeplitz operators, matrix-valued rational functions, Abrahamse's Theorem, subnormal, hyponormal, completion.

\section{REFERENCES}

[1] M. B. ABRahamse, Subnormal Toeplitz operators and functions of bounded type, Duke Math. J. 43 (1976), 597-604.

[2] A. Böttcher and B. Silbermann, Analysis of Toeplitz Operators, Springer, Berlin-Heidelberg, 2006.

[3] C. Cowen, Hyponormality of Toeplitz operators, Proc. Amer. Math. Soc. 103 (1988), 809-812.

[4] C. Cowen And J. Long, Some subnormal Toeplitz operators, J. Reine Angew. Math. 351 (1984), 216-220.

[5] R. E. Curto, I. S. Hwang, D. Kang And W. Y. LeE, Subnormal and quasinormal Toeplitz operators with matrix-valued rational symbols, Adv. Math. 255 (2014), 561-585.

[6] R. E. CURTO, I. S. HwANG AND W. Y. LEe, Hyponormality and subnormality of block Toeplitz operators, Adv. Math. 230 (2012), 2094-2151.

[7] R. E. CURTO, I. S. HWANG AND W. Y. LEE, Which subnormal Toeplitz operators are either normal or analytic?, J. Funct. Anal. 263 (8) (2012), 2333-2354.

[8] R. G. Douglas, Banach algebra techniques in operator theory, Academic Press, New York, 1972.

[9] R. G. Douglas, Banach algebra techniques in the theory of Toeplitz operators, CBMS 15, Providence, Amer. Math. Soc. 1973.

[10] C. FoIAŞ AND A. FRAZHO, The commutant lifting approach to interpolation problems, Operator Theory: Adv. Appl. vol 44, Birkhäuser, Boston, 1993.

[11] C. GU, J. Hendricks AND D. RUtherford, Hyponormality of block Toeplitz operators, Pacific J. Math. 223 (2006), 95-111.

[12] P. R. Halmos, Ten problems in Hilbert space, Bull. Amer. Math. Soc. 76 (1970), 887-933.

[13] P. R. Halmos, Ten years in Hilbert space, Integral Equations Operator Theory 2 (1979), 529-564.

[14] T. NAKAZI AND K. TAKAHASHI, Hyponormal Toeplitz operators and extremal problems of Hardy spaces, Trans. Amer. Math. Soc. 338 (1993), 753-769.

[15] N. K. NiKOLSKII, Treatise on the Shift Operator, Springer, New York, 1986.

[16] V. V. Peller, Hankel Operators and Their Applications, Springer, New York, 2003. 